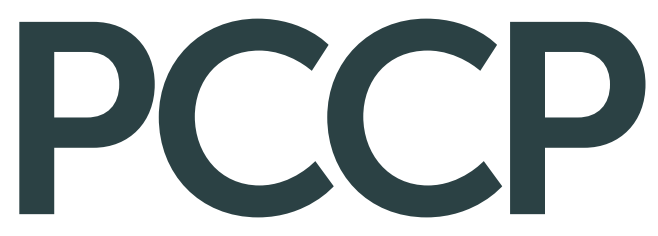

Accepted Manuscript

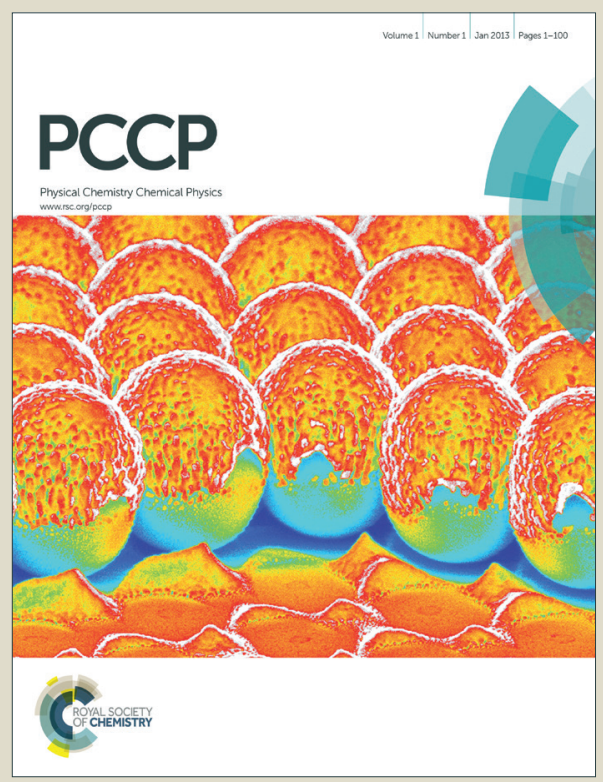

This is an Accepted Manuscript, which has been through the Royal Society of Chemistry peer review process and has been accepted for publication.

Accepted Manuscripts are published online shortly after acceptance, before technical editing, formatting and proof reading. Using this free service, authors can make their results available to the community, in citable form, before we publish the edited article. We will replace this Accepted Manuscript with the edited and formatted Advance Article as soon as it is available.

You can find more information about Accepted Manuscripts in the Information for Authors.

Please note that technical editing may introduce minor changes to the text and/or graphics, which may alter content. The journal's standard Terms \& Conditions and the Ethical guidelines still apply. In no event shall the Royal Society of Chemistry be held responsible for any errors or omissions in this Accepted Manuscript or any consequences arising from the use of any information it contains. 


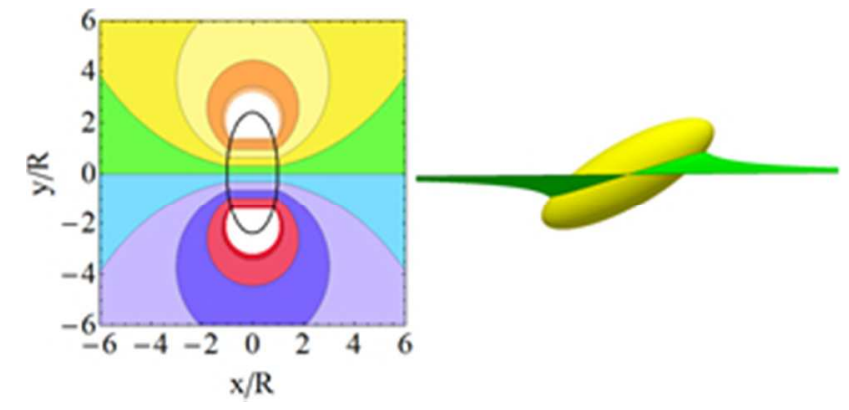

$82 \times 39 m m(96 \times 96$ DPI) 
High accuracy Surface Evolver calculations of the orientational transition for anisotropic magnetic particles at liquid interfaces. 


\title{
Influence of Magnetic Field on the Orientation of Anisotropic Mag- netic Particles at Liquid Interfaces
}

\author{
Bethany J. Newton ${ }^{a}$, Kenneth A. Brakke ${ }^{b}$ and D. Martin A. Buzza ${ }^{* a}$
}

DOI: $10.1039 / b 000000 x$

We study theoretically the influence of an external magnetic field on the orientation of an ellipsoidal magnetic particle adsorbed at a liquid interface. Using the finite element program Surface Evolver, we calculate the equilibrium meniscus shape around the ellipsoidal particle and its equilibrium tilt angle with respect to the undeformed interface $\theta_{t}$ when a magnetic field $B$ is applied perpendicular to the interface. We find that as we increase field strength, $\theta_{t}$ increases and at a critical magnetic field $B_{c 1}$ and tilt angle $\theta_{c 1}$, the particle undergoes a discontinuous transition to the 'perpendicular' orientation $\left(\theta_{t}=90^{\circ}\right)$. Our results agree qualitatively with the simplified theory of Bresme and Faraudo [F. Bresme and J. Faraudo, J. Phys.: Condens. Matter, 2007, 19, 375110] which assumes that the liquid interface is flat, while they agree quantitatively with recent lattice-Boltzmann simulations of Davies et al. [G. Davies et al., Soft Matter, 2014, 10, 6742] which account for the deformation of the liquid meniscus. We also show for the first time that upon reducing the external magnetic field, at a critical magnetic field $B_{c 2}<B_{c 1}$, the particle undergoes a second discontinuous transition from the perpendicular orientation to a finite tilt angle $\theta_{c 2}<\theta_{c 1}$. In other words, for micron-sized particles where the thermal energy $k_{B} T$ is negligible compared to the interfacial energy, the tilt angle vs. magnetic field curve exhibits hysteresis behaviour. Due to the higher degree of accuracy of the Surface Evolver method, we are able to analyse the behaviour of the particles near these orientational transitions accurately and study how the critical quantities $B_{c 1}, B_{c 2}$, $\theta_{c 1}$ and $\theta_{c 2}$ vary with particle aspect ratio and contact angle.

\section{Introduction}

Particles adsorbed at fluid interfaces have been extensively studied in the last three decades due to their many applications in areas ranging from stabilisation of emulsions and foams ${ }^{1}$, nano-structured materials ${ }^{2}$, mineral processing ${ }^{3}$, waste water treatment ${ }^{3}$, personal care products, food and paints ${ }^{4}$. Most of the research in this area has focused on spherical or nearly spherical particles. However, with advances in the synthesis of colloidal particles, particles with other shapes have received increasing attention over the last decade. These shapes include ellipsoids ${ }^{5-7}$, cylinders ${ }^{8-10}$, cubes ${ }^{11}$ and ellipsoidal Janus particles ${ }^{12}$. A contact angle $\theta_{w} \neq 90^{\circ}$ cannot be satisfied around an anisotropic particle by a flat interface, resulting in deformations of the meniscus around the particle ${ }^{5,6,13,14}$. Such deformations lead to long range capillary forces which allow particles to self-organise into a rich variety of structures, which include the particles assembling tip-to-tip and/or side-to-side to form open structures or chains ${ }^{5,15}$. If in addition, we can change the orientation of anisotropic particles by means of an external field, this allows us to tune the capillary interactions between such particles and hence control their self-assembly. For example, recent studies have shown that an external field

${ }^{a}$ Theory of Condensed Matter Group, Department of Physics and Mathematics, University of Hull, Hull HU6 7RX, UK.

${ }^{b}$ Mathematics Department, Susquehanna University, Selinsgrove PA 17870 , USA.

*Corresponding author, E-mail: d.m.buzza@hull.ac.uk can be used to align fibres ${ }^{16}$, induce self-assembled asters ${ }^{17}$ and create switchable 'capillary caterpillars' (long chains of ellipsoidal particles in the side-to-side configuration) ${ }^{18}$. The ability to engineer and control the configuration of anisotropic particles at liquid interfaces opens up exciting possibilities for the manufacture of switchable materials with specific mechanical, optical or magnetic properties.

In this study we investigate theoretically the effect of an external magnetic field on the orientation of a single ellipsoidal particle with a permanent magnetic dipole which is adsorbed at a liquid interface when the magnetic field is applied perpendicular to the interface. In their seminal work, Bresme and Faraudo ${ }^{19}$ and Bresme ${ }^{20}$ analysed this problem using a simple thermodynamic model that assumed that the liquid interface remains planar and that the contact angle of the liquid interface at the particle surface $\theta_{w}=90^{\circ}$ (we will refer to this theory as BF theory). These authors found that at zero field strength the particle has a horizontal orientation (long axis of particle parallel to the interface). However, as the magnetic field is increased, the tilt angle of the particle with respect to the interface gradually increases until, at a critical field strength, the particle undergoes a discontinuous phase transition to the vertical orientation (long axis of particle perpendicular to the interface). These authors also performed molecularly resolved computer simulations of this system and found quantitative agreement with $\mathrm{BF}$ theory across a wide range of field strengths and particle aspect ratios ${ }^{19,20}$. 
However, for a horizontal ellipsoidal particle with contact angle $\theta_{w} \neq 90^{\circ}$ or a tilted ellipsoidal particle of any contact angle, Young's condition of a constant contact angle around the three phase contact line dictates that the liquid meniscus around the particle cannot remain flat. Instead, the liquid meniscus will be deformed with the amplitude of the deformation scaling with particle size. The reason why the effect of such deformations was not observed in the simulations of ref. ${ }^{19,20}$ is presumably because for nanoparticles, the amplitude of the capillary deformations is comparable to the thermal fluctuations of the liquid interface and therefore can be neglected to a first approximation. However, for micron sized particles (which is the focus of this paper) where the amplitude of the capillary deformations is much greater than thermal fluctuations, we expect such deformations to lead to quantitative differences with BF theory. Very recently, Davies et al. have studied this problem for micron-sized ellipsoidal particles with $\theta_{w}=90^{\circ}$ using lattice-Boltzmann simulations ${ }^{21}$ which explicitly account for the deformation of the meniscus. These authors verified that ellipsoidal particles indeed undergo a discontinuous orientational transition with increasing magnetic field. However, they also found significant quantitative differences with BF theory and demonstrated that these differences are due to the deformation of the liquid meniscus.

One limitation of the lattice-Boltzmann method is the fact that the small degree of inherent noise present in the method limits the resolution of the method near the discontinuous transition, which is very sensitive to the presence of any fluctuations in the system. In order to overcome this problem, in this paper we use the finite element package Surface Evolver ${ }^{22}$, which allows us to calculate the equilibrium meniscus around micron-sized particles and analyse the region near the discontinuous transition much more accurately. We also show for the first time that upon reducing the external field, the particle undergoes a second discontinuous transition from the perpendicular orientation to a different tilted state, i.e., we demonstrate that the tilt angle vs. magnetic field curve exhibits a hysteretic behaviour. We furthermore extend the studies in ref. ${ }^{19-21}$ by considering particles with contact angles $\theta_{w} \neq 90^{\circ}$, thus allowing us to study the effect of both particle aspect ratio and contact angle on the orientational transition.

The rest of this paper is organised as follows. In section 2 we discuss the thermodynamics of the problem while in section 3 we provide details of the Surface Evolver method. In section 4 we present our results and discuss the feasibility of observing orientational transitions experimentally in these systems, and finally in section 5 we summarise our main conclusions.

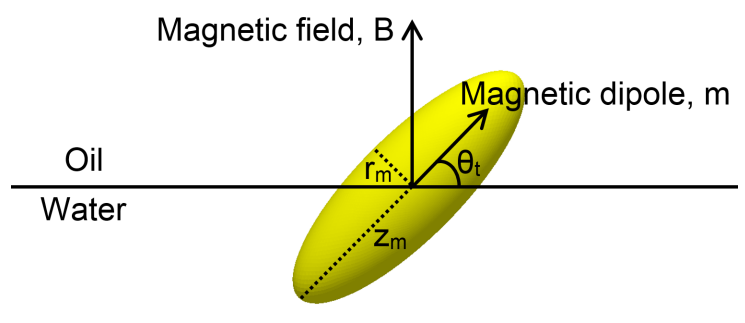

Fig. 1 Geometry of an ellipsoidal particle adsorbed at an oil/water interface in the presence of an external field $B$ applied perpendicular to the interface (for simplicity we show the unperturbed interface). The variables characterising the geometry of the tilted particle are discussed in the main text.

\section{Thermodynamics}

When particles are adsorbed at an interface, the most stable configuration for the particle is the one that removes the maximum area of the liquid interface ${ }^{1}$. This is why, in the absence of an external field, the most stable configuration for an ellipsoidal particle is where the long axis of the particle is parallel to the interface (parallel configuration). This point is obvious if we make the simplifying assumption that the interface around the ellipsoid remains flat ${ }^{19,20}$ but is in fact also true even if we allow for deformations of the liquid interface. Conversely, configurations where the long axis of the particle makes a finite angle to the interface are only stable in the presence of an external field. Let us consider a prolate ellipsoidal magnetic particle adsorbed at a liquid interface, which has a semi-major axis of length $z_{m}$, two semi-minor axes of length $r_{m}$, aspect ratio $\alpha=z_{m} / r_{m}$ and whose long axis makes an angle $\theta_{t}$ with respect to the unperturbed liquid interface (Figure 1). For definiteness, we refer to the upper and lower liquid phases as oil and water respectively. The particle has an embedded magnetic dipole moment $m$ which interacts with an external magnetic field $B$ applied perpendicular to the liquid interface as shown in Figure 1.

The total free energy of this three phase system is given by

$$
F_{\text {int }}=\gamma_{o w} A_{o w}+\gamma_{p o} A_{p o}+\gamma_{p w} A_{p w}-m B \sin \theta_{t}
$$

where $\gamma_{o w}, \gamma_{p o}, \gamma_{p w}$ are the interfacial tensions and $A_{o w}, A_{p o}$, $A_{p w}$ are the areas of the oil/water, particle/oil and particle/water interfaces respectively. Using Young's equation $\gamma_{o w} \cos \theta_{w}=\gamma_{p o}-\gamma_{p w}$ where $\theta_{w}$ is the contact angle of the oil/water interface at the particle surface, noting that $A_{p o}=$ $A_{p}-A_{p w}$ where $A_{p}$ is the total area of the particle and dropping irrelevant constant terms, we can simplify eq. 1 to

$$
F_{\text {int }}=\gamma_{o w} A_{o w}-\gamma_{o w} \cos \theta_{w} A_{p w}-m B \sin \theta_{t}
$$

Finally, it is convenient to divide the above equation through by $\gamma_{o w} A_{p}$ to obtain the dimensionless free energy of the system 
as

$$
\bar{F}_{i n t} \equiv \frac{F_{i n t}}{\gamma_{o w} A_{p}}=\bar{A}_{o w}-\cos \theta_{w} \bar{A}_{p w}-\bar{B} \sin \theta_{t}
$$

where $\bar{A}_{o w}=A_{o w} / A_{p}, \bar{A}_{p w}=A_{p w} / A_{p}$ and $\bar{B}=m B / \gamma_{o w} A_{p}$.

Minimizing $\bar{F}_{\text {int }}$ with respect to $\theta_{t}$ for a given value of $\bar{B}$ allows us to determine the equilibrium tilt angle of the particle for a given magnetic field strength. Note that minimizing $\bar{F}_{\text {int }}$ is equivalent to solving the equation

$$
\frac{1}{\cos \theta_{t}} \frac{\partial \bar{F}_{s t}}{\partial \theta_{t}}=\bar{B}
$$

where

$$
\bar{F}_{s t}=\bar{A}_{o w}-\cos \theta_{w} \bar{A}_{p w}
$$

is the free energy contribution from the interfacial tension terms. Note that the lhs of eq.4 is independent of $\bar{B}$. Thus by calculating the interfacial energy $\bar{F}_{s t}$ and $\frac{\partial \bar{F}_{s t}}{\partial \theta_{t}}$ as a function of $\theta_{t}$, we can determine the equilibrium tilt angle for a given $\bar{B}$ via eq.4.

In order to calculate $\bar{F}_{s t}$, Bresme and Faraudo ${ }^{19}$ made the simplifying assumption that the oil/water interface remains flat in the presence of the adsorbed particle. This allowed them to derive an analytical expression for $\bar{A}_{o w}$ which is given by

$$
\bar{A}_{o w}=\frac{A_{0}}{A_{p}}-\frac{\alpha}{4 G(\alpha)} \sqrt{\frac{1}{\cos ^{2}\left(\theta_{t}\right)+\alpha^{2} \sin ^{2}\left(\theta_{t}\right)}}
$$

where $A_{0}$ is the total area of the unperturbed oil/water interface in the absence of the adsorbed particle and

$$
G(\alpha)=\frac{1}{2}+\frac{1}{2} \frac{\alpha}{\sqrt{1-\alpha^{-2}}} \arcsin \sqrt{1-\alpha^{-2}} .
$$

Bresme and Faraudo further simplified the problem by considering the neutrally wetting case (i.e., $\theta_{w}=90^{\circ}$ ) where the $\bar{A}_{p w}$ term in eq.5 can be neglected. The BF theory predicts a discontinuous transition of the ellipsoidal particle from a finite tilt angle to the perpendicular orientation $\left(\theta_{t}=90^{\circ}\right)$ at a critical field strength. The theory also predicts that the critical field strength increases with increasing particle aspect ratio $\alpha$.

\section{Surface Evolver}

In our study, we calculate both $\bar{A}_{o w}$ and $\bar{A}_{p w}$ numerically using Surface Evolver ${ }^{22}$. This allows us to accurately account for the interfacial deformations caused when analysing the orientational transitions of the particle. Our Surface Evolver model is a finite element method that divides the oil/water interface into a mesh of small triangles; the vertices of these triangles are then displaced to minimise the interfacial energy of the three-phase system. This means that thermal fluctuations are neglected in Surface Evolver. Because of this, the method is accurate for modelling micron-sized particles, where thermal fluctuations are small compared to the amplitude of the meniscus deformation, but is less accurate for modelling nano-sized particles, where thermal fluctuations are comparable to the amplitude of the meniscus deformation.

We define the $x-y$ plane to lie along the unperturbed oilwater interface, the $z$ axis to be perpendicular to the interface and work in length units such that the semi-minor axis length of the particle $r_{m}=1$. In the physical system, the oil/water interface is fixed while the height of the particle relative to the interface is variable depending on the contact angle $\theta_{w}$. In our simulations, this fact is implemented by fixing the centre of the particle at the centre of the simulation cell but allowing the height of the oil/water interface to freely vary relative to the particle, which of course is equivalent to the physical situation. The long axis of the particle is constrained to lie in the $y-z$ plane at an angle of $\theta_{t}$ with respect to the $y$-axis. We use a square simulation cell with side length $12 \times z_{m}$ and impose a fixed contact angle of $\theta_{w}=90^{\circ}$ at the outer edge of the cell. In order to confirm that finite size constraints are negligible, for selected simulations, the simulation cell length was increased by $50 \%$ and yielded essentially the same results for the critical tilt angle (within 2\%) and critical field strength (within $0.1 \%$ ).

The contact angle constraint at the three-phase contact line is imposed by using the edge integral method where the surface integral $A_{p w}$ is partially integrated and represented as a line integral; this eliminates the need to explicitly include the particle/water interface in the calculation ${ }^{22}$. For convenience, all simulation constraints are first represented in the particle reference frame (i.e., with coordinate axes aligned along the major and minor axes of the particle) before being transformed to the $x-y-z$ frame via a coordinate transformation ${ }^{23,24}$. In order to achieve good numerical accuracy, we used a high level of refinement for the oil/water surface, e.g., for particles with an aspect ratio $\alpha=3$, contact angle $\theta_{w}=90^{\circ}$ and tilt angle $\theta_{t}=45^{\circ}$, we used 22500 triangles to represent the surface and 172 vertices to represent the contact line; the specific number of triangles and vertices used was varied depending on the values of $\alpha, \theta_{w}$ and $\theta_{t}$. The minimum-energy surface was found for tilt angles between $0^{\circ}$ and $90^{\circ}$ in increments of $1^{\circ}$. For each tilt angle, we record the location of the contact line and calculate $A_{o w}, A_{p w}$ and hence $\bar{F}_{s t}$ as a function of $\theta_{t}$. The derivative $\frac{\partial \bar{F}_{s t}}{\partial \theta_{t}}$ in eq.4 was then calculated numerically for each simulated tilt angle using the central-difference formula ${ }^{25}$; values of the derivative at other tilt angles were obtained by interpolation.

\section{Results}

We first consider the equilibrium orientation of the ellipsoidal particle as we increase the external field. In Figure 2, we plot 

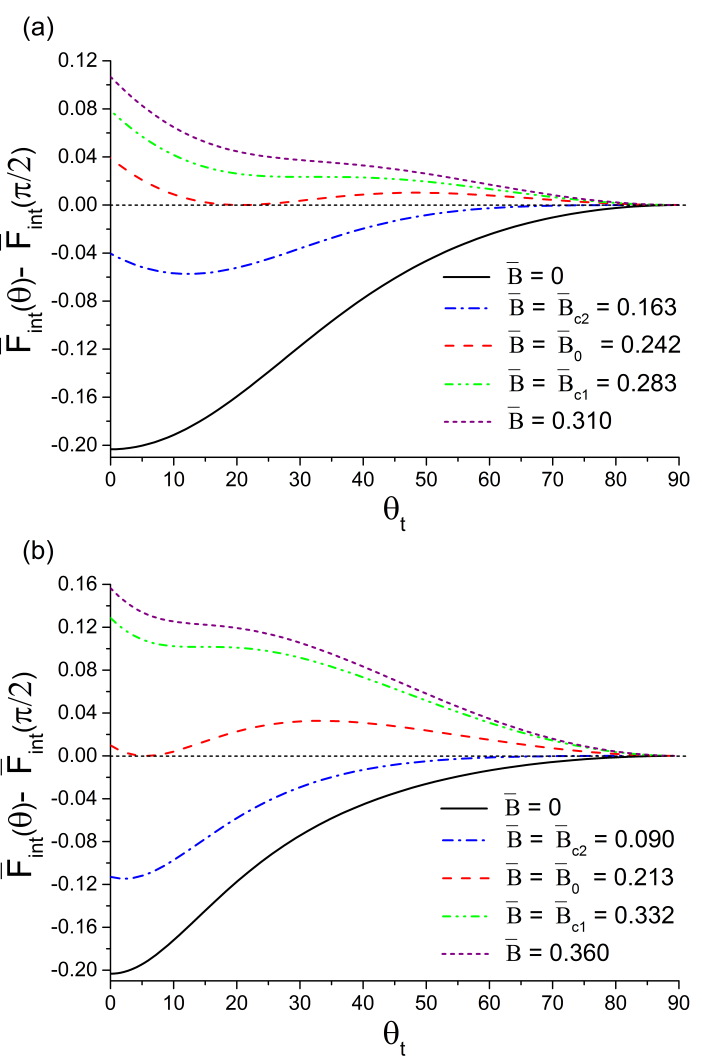

Fig. 2 Dimensionless free energy as a function of tilt angle (relative to perpendicular state) for an ellipsoidal particle with $\alpha=3$, $\theta_{w}=90^{\circ}$ for different field strengths: (a) Surface Evolver results (b) Bresme-Faraudo theory. the total free energy $\bar{F}_{\text {int }}$ given by eq.(3) (relative to the free energy at $\theta_{t}=90^{\circ}$ ) as a function of particle tilt angle $\theta_{t}$ for different field strengths $\bar{B}$ for a particle with aspect ratio $\alpha=3$ and contact angle $\theta_{w}=90^{\circ}$. Figure 2(a), (b) have been calculated using Surface Evolver and BF theory respectively. For each field strength, the equilibrium tilt angle is the one that minimizes the total free energy. For both theories, we see that at zero field, the equilibrium configuration is the 'parallel' state where $\theta_{t}=0^{\circ}$ (black curves).

As we increase the field strength, the equilibrium state becomes the tilted state where the particle has a finite tilt angle that lies between $0^{\circ}<\theta_{t}<90^{\circ}$ (e.g., blue curves). As we increase the field strength further, the free energy curve develops two local minima, one corresponding to the tilted state and the other to the perpendicular state where $\theta_{t}=90^{\circ}$, but the equilibrium state (i.e., global minimum) is still the tilted state. However, at a threshold field strength $\bar{B}_{0}$, the free energy of the tilted state becomes equal to that of the perpendicular state (red curve). At this point, the particle in principle undergoes a first order phase transition from the tilted state to the perpendicular state. However, as first order phase transitions are activated processes, whether this transition can occur in practice depends on the magnitude of the energy barrier between the two local minima relative to the thermal energy $k_{B} T$. For nano-sized particles where the energy barrier is of the order of $k_{B} T$, the first order phase transition can occur and evidence for such a transition has been found in computer simulations of ellipsoidal nanoparticles ${ }^{19,20}$. On the other hand for micronsized particles where the energy barrier is in general thousands of $k_{B} T$ or more, thermal energy is insufficient to activate the first order phase transition and the particle remains trapped in the tilted state for $\bar{B}>\bar{B}_{0}$, even though the tilted state is no longer the equilibrium state (i.e., it is a metastable state). Finally, as we increase the field strength further, at a critical field $\bar{B}_{c 1}$, the local minimum corresponding to the tilted state merges with the local maximum corresponding to the free energy barrier at the critical tilt angle $\theta_{c 1}$ (green curve). At this point, the energy barrier disappears and the particle undergoes an irreversible transition from the tilted state to the perpendicular state.

Comparing Figure 2(a) and (b), we see that both Surface Evolver and BF predict the same qualitative features for the orientational transition. However, there are clearly significant quantitative differences between Surface Evolver and BF theory. These differences are illustrated more clearly in Figure 3 where we plot the equilibrium tilt angle $\theta_{t}$ as a function of the external field $\bar{B}$ for increasing fields for $\theta_{w}=90^{\circ}$ and $\alpha=1.5$ or $\alpha=3$. Specifically, we compare the results for Surface Evolver, BF theory and the recent lattice-Boltzmann simulations of Davies et al. ${ }^{21}$, which explicitly account for the deformation of the liquid meniscus around the particle. Comparing first of all Surface Evolver and BF theory, we see that both 


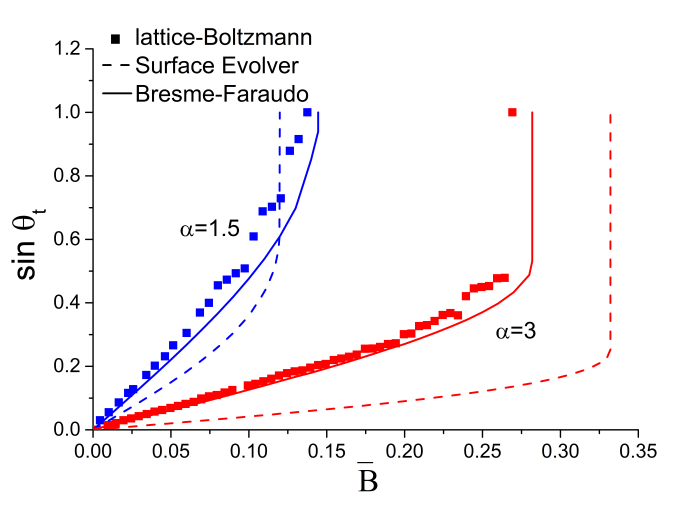

Fig. 3 Equilibrium tilt angle as a function of dimensionless field strength for increasing fields calculated using Bresme-Faraudo theory (solid line), Surface Evolver (dashed line) and lattice-Boltzmann simulations ${ }^{21}$ (points) for a contact angle $\theta_{w}=90^{\circ}$ and two different aspect ratios $\alpha=1.5$ (blue) and $\alpha=3$ (red).

theories agree qualitatively and predict that the particle undergoes a discontinuous orientation transition above a critical field strength. However, there are clearly significant quantitative differences between BF theory and Surface Evolver. For example for $\alpha=1.5$, Surface Evolver predicts a larger critical field $\bar{B}_{c 1}$ and larger critical tilt angle $\theta_{c 1}$ compared to BF theory, while for $\alpha=3$, Surface Evolver predicts a smaller critical field and larger critical tilt angle compared to BF theory. The results of Figures 2 and 3 demonstrate that assuming a flat fluid interface allows us to capture the essential qualitative features of the orientational transition. However, if we want to obtain quantitative results for the orientational behaviour of micron-sized anisotropic particles, we need to explicitly account for the deformation of the interface.

Next we compare Surface Evolver with the latticeBoltzmann simulations in Figure 3. We see that for both $\alpha=1.5,3$, there is excellent quantitative agreement between the two theories when we are far enough away from the orientational transition. However, discrepancies between the two theories begin to appear near the orientational transition where the lattice-Boltzmann results become noisy. We believe that these discrepancies are due to the small degree of noise that is inherent in the lattice-Boltzmann method. While this noise does not have a significant effect when we are far enough away from the orientational transition, it has a big impact near the discontinuous transition, which is very sensitive to any fluctuations in the system. These results illustrate the necessity of very accurate numerics if we want to capture the behaviour near the orientational transition accurately. In this context, Surface Evolver complements the lattice-Boltzmann scheme and allows us to analyse the region near the orientational transition to a much higher degree of resolution.

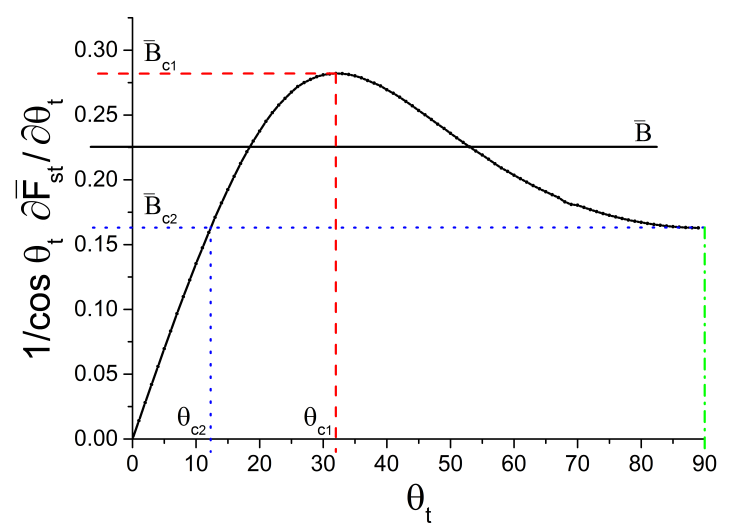

Fig. $4 \frac{1}{\cos \theta_{t}} \frac{\partial \bar{F}_{s t}}{\partial \theta_{t}}$ as a function of tilt angle $\theta_{t}\left(\bar{F}_{s t}\right.$ is the dimensionless interfacial tension free energy of the system) for an ellipsoidal particle with $\alpha=3, \theta_{w}=90^{\circ}$. The equilibrium tilt angle for a given external field $\bar{B}$ (represented by the solid horizontal line) is given by the intersection of the horizontal line with the rising part of the curve. The values of the critical fields and tilt angles can be determined from the curve as shown above.

One very important feature for micron-sized particles that has not been discussed previously is the fact that the very large energy barrier between local minima states for such particles implies that there will be significant hysteresis in their orientational behaviour. This can be seen by analysing Figure 2(a) or (b) for the reverse case where we decrease the external field. For high external fields, the equilibrium state is the perpendicular state (e.g., purple curve). However, as we decrease the external field to less than $\bar{B}_{c 1}$, the free energy curve develops two local minima, one corresponding to the perpendicular state and the other to the tilted state where $0^{\circ}<\theta_{t}<90^{\circ}$, but the equilibrium state (i.e., global minimum) is still the perpendicular state. However, at the threshold field strength $\bar{B}_{0}$, the free energy of the tilted state becomes equal to that of the perpendicular state (red curve). At this point, the particle should undergo a first order phase transition from the perpendicular state to the tilted state. However, the very large energy barrier between the two states prevents the particle from doing so and it remains trapped in the (now metastable) perpendicular state for $\bar{B}<\bar{B}_{0}$. Finally, as we decrease the field strength further, at a critical field $\bar{B}_{c 2}$, the local maximum corresponding to the free energy barrier merges with the local minimum corresponding to the perpendicular state (blue curve). At this point, the energy barrier disappears and the particle undergoes an irreversible transition from the perpendicular state to the tilted state with tilt angle $\theta_{c 2}<\theta_{c 1}$. As can be seen from Figure 2(b), this second irreversible transition is also predicted by $\mathrm{BF}$ theory. However, as far as we are aware, the presence of hysteresis in the orientational transition of ellipsoidal magnetic particles at a liquid interface has not to date been discussed 
explicitly in the literature. We emphasize that we only expect such hysteretic behaviour to be seen for micron sized particles where the activation energy is large. For nano-sized particles, where the activation energy is small (order $k_{B} T$ or less) ${ }^{19,20}$, we expect this hysteretic behaviour to disappear and the orientational transition to occur via an equilibrium first order transition.

Numerically, we have found that a convenient method for determining the equilibrium tilt angle, the critical fields $\bar{B}_{c 1}$, $\bar{B}_{c 2}$ and the critical tilt angles $\theta_{c 1}, \theta_{c 2}$ is by solving eq.4. This is illustrated in Figure 4 where we plot the curve $\frac{1}{\cos \theta_{t}} \frac{\partial \bar{F}_{s t}}{\partial \theta_{t}}$ as a function of $\theta_{t}$ for $\alpha=3, \theta_{w}=90^{\circ}$. For an arbitrary magnetic field $\bar{B}$, represented by the solid horizontal line in Figure 4, the intersection with the rising part of the curve represents the local minimum of the free energy curve corresponding to the tilted state; the value of $\theta_{t}$ at the intersection is therefore the equilibrium tilt angle. The intersection of the horizontal line with the falling part of the curve represents the local maximum of the free energy curve corresponding to the energy barrier (see Figure 2). The first irreversible transition occurs when the external field is such that the tilted state merges with the energy barrier which corresponds to the maximum of the curve in Figure 4. We can therefore determine $\bar{B}_{c 1}$ and $\theta_{c 1}$ from the magnitude and position of the maximum, as shown in Figure 4. On the other hand, the second irreversible transition occurs when the external field is such that energy barrier merges with the local minimum at $\theta_{t}=90^{\circ}$. We can therefore determine $\bar{B}_{c 2}$ from the value of the curve at $\theta_{t}=90^{\circ}$; the intersection of $\bar{B}_{c 2}$ with the rising part of the curve then yields $\theta_{c 2}$ as shown in Figure 4.

In Figure 5, we plot the equilibrium tilt angle as a function of magnetic field for both increasing fields (lower curve) and decreasing fields (upper curve) for $\alpha=3, \theta_{w}=90^{\circ}$; (a) and (b) are calculated using Surface Evolver and BF theory respectively. The position of the irreversible orientational transitions at $\bar{B}_{c 1}$ and $\bar{B}_{c 2}$ are indicated on the plot. The position of the threshold field $\bar{B}_{0}$ where a reversible first order phase transition can occur (for particles with sufficiently large activation energy) is also indicated. Note that the lower curve is metastable for $\bar{B}_{0}<\bar{B}<\bar{B}_{c 1}$ while the upper curve is metastable for $\bar{B}_{c 2}<\bar{B}<\bar{B}_{0}$. Once again we see that both Surface Evolver and BF theory agree qualitatively, predicting that there is a significant degree of hysteresis in the orientational transition of the particle. However, because of the different assumptions regarding the deformation of the meniscus, there are clearly significant quantitative differences between the two: firstly Surface Evolver predicts a much narrower hysteresis loop compared to BF theory; secondly the critical tilt angles predicted by Surface Evolver are significantly higher than the corresponding tilt angles predicted by BF theory.

Given the importance of the deformation of the liquid meniscus for quantitative calculations of the orientational tran-
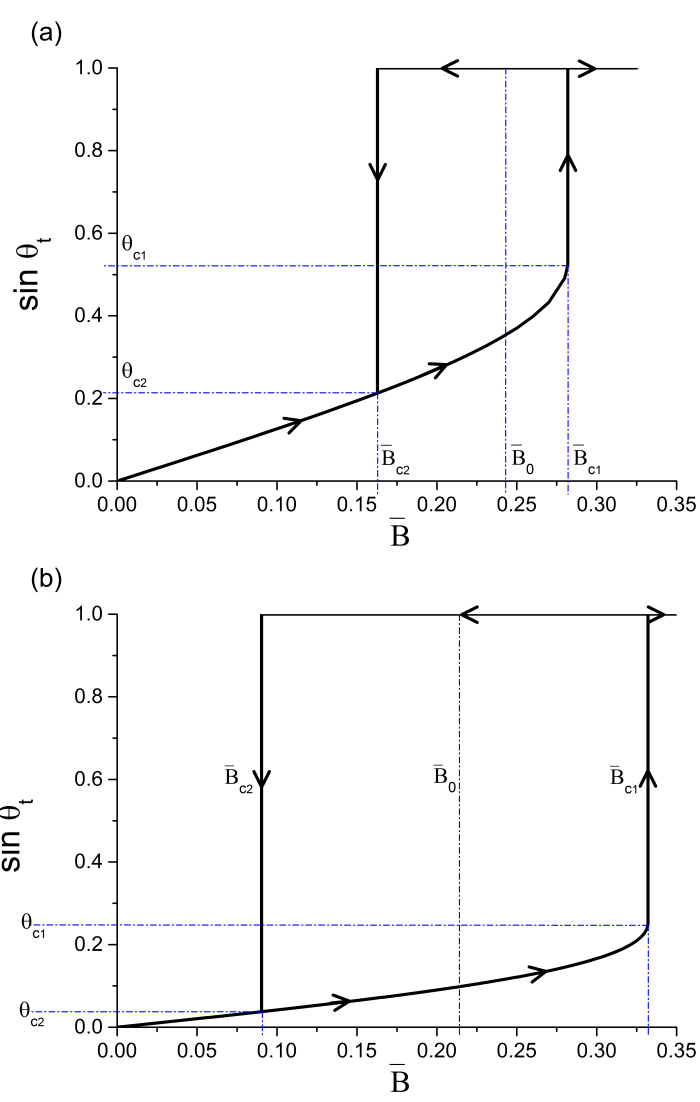

Fig. 5 Hysteresis curve for the equilibrium tilt angle vs. dimensionless field strength for increasing and decreasing fields (as indicated by the direction of the arrows) for $\alpha=3, \theta_{w}=90^{\circ}$ calculated using: (a) Surface Evolver (b) Bresme-Faraudo theory. 
(a)
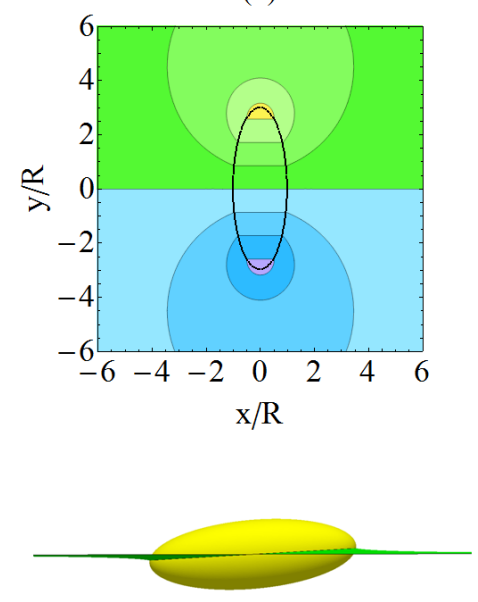

(b)
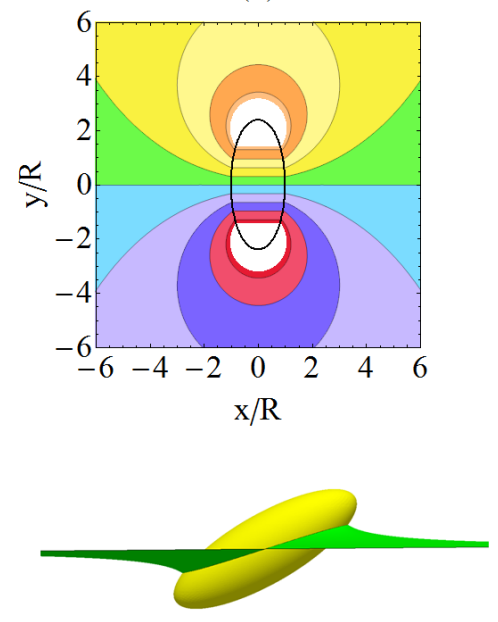

(c)
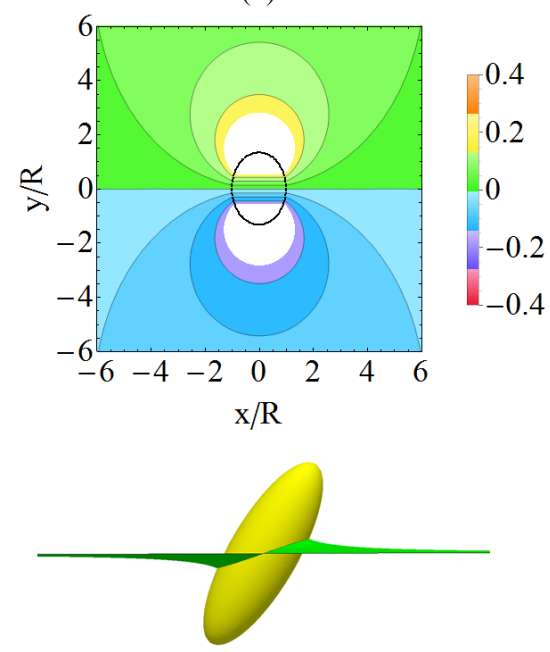

Fig. 6 Contour plot (top) and 3D plot (bottom) of the deformation field for the oil/water interface calculated from Surface Evolver for three different tilt angles $\theta_{t}$ of a particle with $\alpha=3, \theta_{w}=90^{\circ}$ : (a) $\theta_{t}=5^{\circ}$ (b) $\theta_{t}=30^{\circ}$ (c) $\theta_{t}=60^{\circ}$.

sition $^{21}$, it is instructive to analyse the deformation of the liquid meniscus around the particle as a function of the tilt angle using Surface Evolver. In Figure 6, we plot the deformation field of the oil/water interface for a particle with $\alpha=3$ and $\theta_{w}=90^{\circ}$ for some representative tilt angles as contour plots (top) and 3D plots (bottom); the solid oval outline in the contour plots represent the projection of the three-phase contour line onto the $x-y$ plane. We have chosen a contact angle of $\theta_{w}=90^{\circ}$ for clarity since for this neutral wetting condition, any quadrupolar deformations due to contact angle constraints ${ }^{5-7}$ are absent. The deformation field is clearly dipolar in nature, in agreement with the lattice-Boltzmann simulations of Davies et al. ${ }^{21}$. We also note that the deformation is small for small (a) and large (c) tilt angles and is maximum for intermediate tilt angles (b). This is not surprising since (for $\theta_{w}=90^{\circ}$ ) the deformation is zero for $\theta_{t}=0^{\circ}$ and $90^{\circ}$. Interestingly the tilt angle at which the maximum deformation occurs $\left(\approx 30^{\circ}\right.$ in this case, i.e., case (b)) is essentially equal to $\theta_{c 1}$, the critical angle for the irreversible transition to the perpendicular state to occur. Qualitatively this can be understood from the fact that the maximum deformation effectively corresponds to the maximum torque that can be generated by interfacial tension to oppose the magnetic torque. Increasing the tilt angle beyond this point leads to a further increase in the magnetic torque but a decrease in the interfacial tension torque and the particle therefore undergoes a discontinuous transition to the perpendicular state.

In Figure 7, we analyse the dependence of the critical fields and critical tilt angles on the aspect ratio of the particles $\alpha$. Specifically, in Figure 7(a), we plot $\theta_{c 1}$ and $\theta_{c 2}$ as a function of $\alpha$ while in Figure 7(b) we plot $\bar{B}_{c 1}$ and $\bar{B}_{c 2}$ as a function (a)

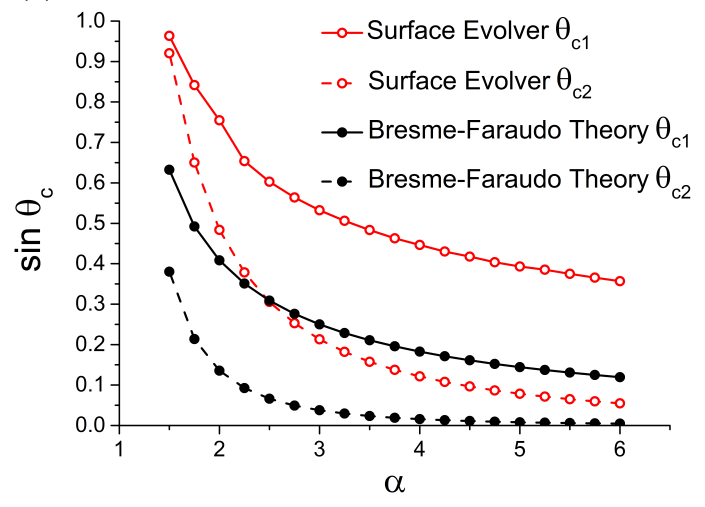

(b)

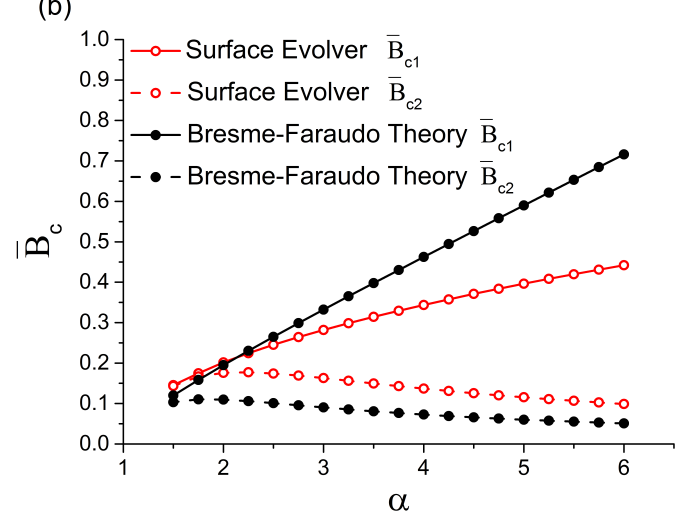

Fig. 7 (a) Critical tilt angles $\theta_{c 1}, \theta_{c 2}$ and (b) critical field strengths $\bar{B}_{c 1}, \bar{B}_{c 2}$ as a function of aspect ratio $\alpha$ for a particle with $\theta_{w}=90^{\circ}$ calculated using Surface Evolver and Bresme-Faraudo theory. 
of $\alpha$ for $\theta_{w}=90^{\circ}$; the red lines are the predictions of Surface Evolver while the black lines are the predictions of BF theory. We see that BF theory agrees qualitatively with Surface Evolver. Specifically, both theories predict that $\theta_{c 1}, \theta_{c 2}$ decrease with increasing $\alpha$ and the width of the hysteresis curve $\bar{B}_{c 1}-\bar{B}_{c 2}$ increases with increasing $\alpha$. Interestingly, for an aspect ratio of $\alpha=1.5$, the width of the hysteresis curve falls to practically zero for both Surface Evolver and BF theory. However, as already noted in Figure 5, Surface Evolver predicts significantly higher critical tilt angles compared to BF theory for any given aspect ratio $\alpha$ (Figure 7(a)) and a significantly narrower width for the hysteresis curve compared to BF theory for any given $\alpha$ (Figure 7(b)).

In Figure 8, we use Surface Evolver to analyse the dependence of the critical fields and critical tilt angles on the contact angle of the particles $\theta_{w}$. This represents an extension to BF theory ${ }^{19,20}$ and ref. ${ }^{21}$ which were restricted to the neutral wetting condition $\theta_{w}=90^{\circ}$. Specifically, in Figure 8(a), we plot $\theta_{c 1}$ and $\theta_{c 2}$ as a function of $\theta_{w}$ while in Figure $8(\mathrm{~b})$ we plot $\bar{B}_{c 1}$ and $\bar{B}_{c 2}$ as a function of $\theta_{w}$ for $\alpha=3$. We see that for increasing contact angle away from $90^{\circ}$, both the critical tilt angle and the critical field strength decrease. This makes physical sense since for increasing contact angle, more of the particle enters the oil phase, thus reducing the area of the oil/water interface removed by the particle. This reduces the interfacial tension torque relative to the magnetic torque acting on the particle, resulting in a decrease for both the tilt angle and field strength needed for orientational transitions of the particle.

Finally, we consider the feasibility of observing the above orientational transitions experimentally. Firstly, for a typical micron-sized system possessing a permanent magnetic dipole, we use parameters for anisotropic maghemite $\left(\gamma-\mathrm{Fe}_{2} \mathrm{O}_{3}\right)$ particles ${ }^{26}$ prepared by the group of Paul Clegg at Edinburgh ${ }^{27}$. Assuming typical rod lengths of $L=3 \mu \mathrm{m}$ and aspect ratios of $\alpha=10$, this yields a magnetic dipole moment $m=$ $4 \times 10^{-14} \mathrm{~A} \cdot \mathrm{m}^{-2}$. Assuming a contact angle of $\theta_{w}=90^{\circ}$, for $\alpha=10$ the dimensionless critical field for the tilt to perpendicular transition is $\bar{B}_{c 1} \approx 0.5$ (by extrapolating Figure 7). Using a typical oil/water tension of $\gamma_{o w}=30 \mathrm{mN} \cdot \mathrm{m}^{-1}$, this translates to a real magnetic field of $B=0.7 \mathrm{~T}$, which is achievable experimentally.

Next, for a typical micron-sized paramagnetic system, we use the parameters considered in ref. ${ }^{19}$ with rod length $L=$ $3 \mu \mathrm{m}$, aspect ratio $\alpha=1.7$, oil $/$ water tension $\gamma_{o w}=10 \mathrm{mN} \cdot \mathrm{m}^{-1}$ and magnetic susceptibility $\chi=10$. We further assume that the magnetic dipole is given by $m=\chi B / \mu_{0} \cdot \pi d^{2} L / 4$, where $B$ is the external magnetic field, $\mu_{0}$ is the permeability of free space and $d$ is the diameter of the rod. Assuming a contact angle of $\theta_{w}=90^{\circ}$, for $\alpha=1.7$ we have $\bar{B}_{c 1} \approx 0.1$, which translates to a real magnetic field of $B=0.02 \mathrm{~T}$. This is in excellent agreement with the estimate in ref. ${ }^{19}$ and is easily achievable experimentally.
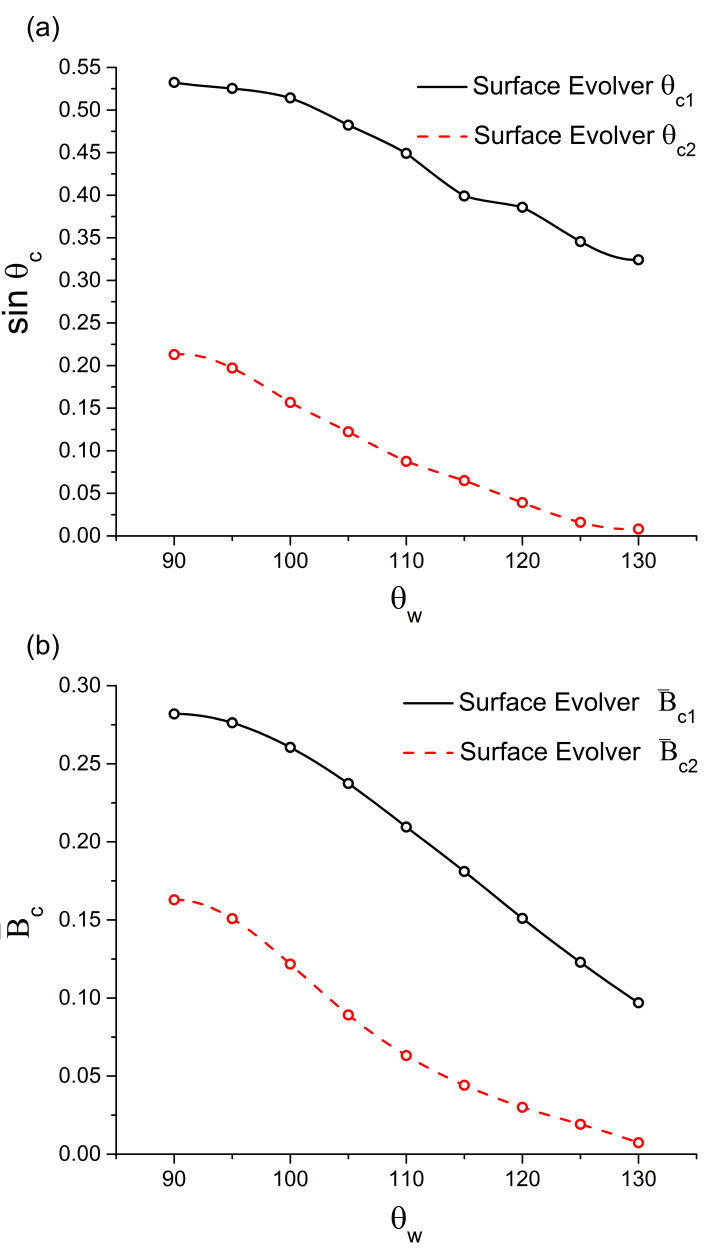

Fig. 8 (a) Critical tilt angles $\theta_{c 1}, \theta_{c 2}$ and (b) critical field strengths $\bar{B}_{c 1}, \bar{B}_{c 2}$ as a function of contact angle $\theta_{w}$ (in degrees) for a particle with aspect ratio $\alpha=3$ calculated using Surface Evolver. 
From Figures 7 and 8, we note that these critical fields can be readily tuned by a factor of up to 3 to 4 by changing particle aspect ratio or contact angle within a reasonable range. Interfacial magnetic ellipsoids are therefore a versatile system whose properties can be readily tailored for specific applications.

\section{Conclusions}

Using the finite element package Surface Evolver, we have studied the orientational transitions of an ellipsoidal magnetic particle adsorbed at a liquid interface due to an applied external field, explicitly accounting for the deformation of the liquid meniscus around a particle. We find that when the magnetic field is increased beyond a critical field $\bar{B}_{c 1}$, the particles undergo a discontinuous transition to the perpendicular state (tilt angle $\theta_{t}=90^{\circ}$ ). Our results are in qualitative agreement with the simplified model of Bresme and Faraudo ${ }^{19,20}$ (which assume a flat liquid interface) and in quantitative agreement with recent lattice-Boltzmann simulations ${ }^{21}$ (which account for deformation of the liquid interface). Our calculations demonstrate that whilst assuming a flat interface allows us to capture the essential qualitative features of the orientational transition, it is important to explicitly include the deformation of the liquid interface for quantitative calculations of the transition. We also show that there is significant hysteresis in the orientational transition of micron-sized ellipsoidal particles due to the very large energy barriers that exist between the tilted and perpendicular states for this system. This hysteresis is in fact also predicted by the model of Bresme and Faraudo but has not been explicitly discussed previously. For currently available micron-sized anisotropic magnetic particles, we show that the critical magnetic fields required to induce the orientational phase transitions discussed above are achievable experimentally. Furthermore, we demonstrate that these critical fields can be readily tuned by a factor of 3 to 4 by changing the aspect ratio or contact angle of the magnetic particles. This interfacial system therefore represents a versatile platform which can be used to design switchable materials with specific mechanical, optical or magnetic properties.

\section{Acknowledgments}

BJN is grateful to The University of Hull, The Joseph and Annie Cattle Trust, The Elizabeth Walker Foundation and The Ann Watson's Trust for financial support. We also acknowledge useful discussions with Gary Davies.

\section{References}

1 B. P. Binks, Curr. Opin. Colloid Interface Sci., 2002, 7, 21.

2 K. P. Velikov and O. D. Velev, Colloidal Particles at Fluid Interfaces, Cambridge Univ. Press, 2006, p. 225.

3 A. V. Nguyen, R. J. Pugh and G. J. Jameson, Colloidal Particles at Fluid Interfaces, Cambridge Univ. Press, 2006, p. 328.

4 P. A. Kralchevsky and K. Nagayama, Particles at Fluid Interfaces and Membranes: Attachment of Colloid Particles and Proteins to Interfaces and Formations of TwoDimensional Arryas, Elsevier, Amsterdam, 2001, p. vi.

5 J. C. Loudet, A. M. Alsayed, J. Zhang and A. G. Yodh, Phys. Rev. Lett., 2005, 94, 018301.

6 J. C. Loudet, A. G. Yodh and B. Pouligny, Phys. Rev. Lett., 2006, 97, 018304.

7 H. Lehle, E. Noruzifar and M. Oettel, Eur. Phys. J. E, 2008, 26, 151.

8 E. P. Lewandowski, J. A. Bernate, P. C. Searson and K. J. Stebe, Langmuir, 2008, 24, 9302.

9 E. P. Lewandowski, J. A. Bernate, A. Tseng, P. C. Searson and K. J. Stebe, Soft Matter, 2009, 5, 886.

10 E. P. Lewandowski, M. Cavallaro, Jr., L. Botto, J. C. Bernate, V. Garbin and K. J. Stebe, Langmuir, 2010, 26, 15142.

11 G. Morris, S. J. Neethling and J. J. Cilliers, Miner. Eng., 2010, 23, 979.

12 B. J. Park and D. Lee, ACS Nano, 2012, 6, 782.

13 P. A. Kralchevsky and K. Nagayama, Adv. Colloid Interface Sci., 2000, 85, 145.

14 J. C. Loudet and B. Pouligny, Europhys. Lett., 2009, 85, 28003.

15 L. Botto, E. P. Lewandoski, M. Cavallaro, Jr. and K. J. Stebe, Soft Matter, 2012, 8, 9957.

16 T. Kimura, M. Yoshino, T. Yamane, M. Yamato and M. Tobita, Langmuir, 2004, 20, 5669.

17 A. Snezhko and I. S. Aranson, Nat. Mater, 2011, 10, 698.

18 G. B. Davies, T. Kruegar, P. V. Coveney, J. Harting and F. Bresme, Adv. Mater, 2014 DOI: 10.1002/adma.201402419.

19 F. Bresme and J. Faraudo, J. Phys.: Condens. Matter, 2007, 19, 375110.

20 F. Bresme, Eur. Phys. J. B, 2008, 64, 487.

21 G. Davies, T. Kruegar, P. Coveney, J. Harting and F. Bresme, Soft Matter, 2014, 2014, 10, 6742.

22 K. A. Brakke, Exp. Math., 1992, 1, 141.

23 C. Raufaste, G. Kirstetter, F. Celestini and S. J. Cox, Europhys. Lett., 2012, 99, 24001.

24 C. Raufaste and S. J. Cox, Colloids Surf., A, 2013, 438, 
126.

25 K. F. Riley, M. P. Hobson and S. J. Bence, Mathematical Methods for Physics and Engineering, 2nd Ed., Cambridge Univ. Press, 2002, p. 1179.

26 P. Mendoza-Zelis, D. Muraca, J. S. Gonzalez, G. A. Pasquevich, V. A. Alvarez, K. R. Pirota and F. H. Snchez, J. Nanopart. Res., 2013, 15, 1613.

27 Niek Hijnen, PhD Thesis, University of Edinburgh, 2013, Chapter 4. 